\title{
Whole Genome Association Study
}

National Cancer Institute

\section{Source}

National Cancer Institute. Whole Genome Association Study. NCI Thesaurus. Code C93020.

A study that looks at the genetic variations found in individuals to determine if either a variant or a set of variants is associated with a trait, biological process or disease. 